\title{
LAS ACTITUDES Y EL GRADO DE IDENTIDAD HACIA EL ZAPOTECO POR SUS HABLANTES EN UN NUEVO ESCENARIO DE LLEGADA: ENSENADA, BAJA CALIFORNIA, MÉXICO ${ }^{1}$
}

\author{
Eyder Gabriel Sima Lozano \\ Universidad Autónoma de Baja California (México) \\ eyder.sima@uabc.edu.mx \\ Jesús Eduardo Fong Flores \\ Universidad Autónoma de Baja California (México) \\ jfong@uabc.edu.mx \\ Carlos Ivanhoe Gil Burgoin \\ Universidad Autónoma de Baja California (México) \\ cgil@uabc.edu.mx
}

Recibido: 07/02/2020 - Aprobado: 27/03/2020

DOI: doi.org/10.17533/udea.lyl.n78a09

\begin{abstract}
Resumen: La presente investigación tiene como objetivo identificar actitudes lingüísticas hacia el zapoteco y la construcción de su identidad por sus hablantes en la ciudad de Ensenada (Baja California, México) considerando que existen pocas investigaciones sobre esta lengua desde la sociolingüística en este escenario. A partir de una metodología cualitativa, con un análisis inductivo, usando un cuestionario sociolingüístico y diario de campo, el estudio obtuvo los testimonios de 10 hablantes zapotecos de la zona. Los informantes revelaron actitudes positivas hacia su lengua materna y la continuidad de su identidad como zapotecos motivada por su lugar de origen: San Blas (Oaxaca).
\end{abstract}

Palabras clave: actitudes; identidad; migración; zapoteco; Ensenada.

1. Este trabajo es resultado del proyecto titulado Actitudes lingüísticas de la población indígena migrante hacia sus lenguas vernáculas, en algunos escenarios urbanos de Baja California, con financiamiento de la $20^{\mathrm{a}}$ Convocatoria Interna de Apoyo a Proyectos de Investigación de la Universidad Autónoma de Baja California (México).

LINGÜÍSTICA Y LITERATURA 


\title{
THE ATTITUDES AND LEVEL OF IDENTITY TOWARDS THE ZAPOTEC LANGUAGE BY ITS SPEAKERS IN A NEW ARRIVAL SCENARIO: ENSENADA, BAJA CALIFORNIA, MEXICO
}

\begin{abstract}
This research aimed to identify linguistic attitudes towards the Zapotec and the construction of its identity by its speakers in the city of Ensenada (Baja California, Mexico), whereas there is little sociolinguistic research on this language in this scenario. Based on a qualitative methodology, with inductive analysis, using a sociolinguistic questionnaire and field diary, the study obtained the testimonies of 10 Zapotec speakers from the area. The informants revealed positive attitudes towards their mother language and the continuity of their identity as Zapotecs motivated by their place of origin: San Blas (Oaxaca).
\end{abstract}

Key words: attitudes; identity; migration; Zapotec language; Ensenada.

\section{Introducción}

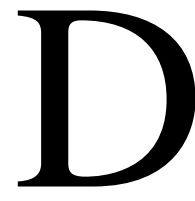
esde la sociolingüística (Barriga \& Parodi, 1999) los estudios de actitudes tienen relevancia para el análisis de la vitalidad, los procesos de mantenimiento y desplazamiento. En ellas se encuentran saberes, emociones y reacciones ante el idioma, por lo que funcionan como punto de partida para el conocimiento de la situación de una lengua. En esta perspectiva, el presente trabajo incidió en el análisis de las actitudes hacia una lengua indígena por sus propios hablantes, como lo es el zapoteco, en una situación de migración en la ciudad de Ensenada, escenario en el que indígenas provenientes del sur de México se desempeñan en actividades predominantemente agrícolas.

Por otro lado, las actitudes linguiísticas contribuyen a los estudios de migración e identidad, pues los hablantes no solo se trasladan a un nuevo espacio de llegada, sino que también configuran nuevas relaciones hacia su lengua en los nuevos territorios a los que arriban. De esta forma, entender la situación de una lengua a través de las actitudes lingüísticas, conlleva a comprender el proceso migratorio de los hablantes, quienes realizan procesos de mantenimiento o desplazamiento del idioma a partir de las presiones del contexto con el que entran en contacto (Terborg \& García, 2011).

En la literatura reportada sobre estudios de actitudes lingüísticas y en relación con el tema objeto de este estudio, se encontraron algunos ejemplos de corte nacional como el trabajo de Sordo (2019), quien analizó las actitudes y la identidad étnica de indígenas 
migrantes en la zona metropolitana de Monterrey, al encontrar posiciones discrepantes hacia la lengua, como las fuertes presiones para el favorecimiento de la identidad indígena.

Por su parte, Álvarez (2008), observó a través del discurso las actitudes hacia la lengua maya en la comunidad de Holcá, detectando actitudes no siempre positivas hacia la lengua. Simultáneamente, Terborg y Velázquez (2008), examinaron las percepciones de universitarios de antropología en la Universidad Autónoma del Estado de Morelos en Toluca, pero éstas no fueron favorables hacia la variante del español con acento indígena. Un año antes, Castillo (2007), indagó sobre las posturas hacia el náhuatl en Cuetzalan, (Puebla) y determinó que si bien existe un alto valor hacia la lengua que sus hablantes llaman mexicano, suelen despreciar la variante mezclada con el español.

En la investigación de Durán y Sauma (2003), se exploraron las actitudes lingüísticas en las escuelas primarias, secundarias y facultades de la ciudad de Mérida, donde se ha llevado a cabo la implementación de la lengua maya como materia optativa y experimental, donde se identificaron desventajas hacia la lengua, solo que con un promedio general de aceptación por parte de los estudiantes. En un estudio de corte rural (Pfeiler, 1993) se expusieron las condiciones sociales de la población rural y de los bilingües de maya y español, como las que dificultaron la aplicación de la prueba del matched-guise en dos comunidades mayas. A partir de dicho estudio, se concluyó que las actitudes resultaron propicias en la mayoría de los predicados evaluados hacia el español en comparación con el maya.

En el ámbito internacional puede mencionarse el análisis de las actitudes hacia el español por parte de familias mexicanas y colombianas (Vargas, 2018). Al respecto, se destacaron resultados positivos hacia el español con motivaciones diferenciadas entre los dos grupos. Por su parte, Song y Wang (2017), abordaron las actitudes de estudiantes chinos hacia cuatro variantes del español: el castellano, mexicano, colombiano y argentino, concluyendo que la variante de España, debido a las políticas lingüísticas y el contacto amplio entre España y China, es la preferida por el sector de población estudiado. En tanto, Gooskens, Hilton y Anja (2016), investigaron sobre las actitudes hacia el sueco y el danés por parte de comunidades extranjeras de los dos países, en las que no se encontraron diferencias significativas hacia una lengua y otra, pues los participantes del estudio no 
estaban influenciados por las visiones y normas sociales a diferencia de los escandinavos nativos.

Hotařová (2011), se centró propiamente no en una comunidad, sino en el instrumento para el estudio de actitudes conocido como matched-guise o la técnica de pares ocultos. Describió su aplicación en España, sobre todo en la comunidad valenciana, y presentó una serie de autores muy representativos que habían realizado estudios usando el instrumento, por lo que el trabajo fue una excelente fuente tanto para la revisión de la literatura como para la metodología. En este mismo tenor, Solís (2002), comparó los pros y contras de la técnica del matched-guise, al presentar las soluciones de las desventajas desde la postura del mismo Lambert y haciendo énfasis en que la técnica debe realizar grabaciones centradas en experiencias reales que experimenten los hablantes, a fin de que éstos sientan familiaridad con el contenido de las grabaciones. Finalmente, no se puede dejar de mencionar a Lambert (1972), pionero de las actitudes lingüísticas, quien creó la técnica del matched-guise, al aplicar el instrumento en una población de habla francesa e inglesa, dando como resultado una predominancia de las actitudes hacia el inglés, aun en una comunidad de origen francófono.

Cabe revisar los estudios sociolingüísticos hacia las lenguas indígenas del contexto inmediato a este estudio, del cual únicamente se encontró el trabajo de Crhová (2004), el cual trata sobre un estudio de actitudes por la población de Tijuana hacia las lenguas indígenas, el español y sus variantes, así como el inglés. En el caso de las lenguas indígenas la autora centró su atención particular en la extinción de algunas lenguas nativas de Baja California, tales como el paipai, el kiliwa, kumiai o el cucapá. Otros estudios tienen relación con la vitalidad, situación sociolingüística, adquisición de la escritura de la lengua, educación en el aula de clases y migración, destacando a Sánchez y Rojas (2016), quienes estudiaron el paipai en la comunidad de Santa Catarina, donde se estableció que únicamente los hablantes entre los 46 y 65 años presentan un grado de vitalismo aceptable, ya que el español es la lengua más usada por las nuevas generaciones. En ese sentido, Leyva (2016), reportó números negativos de los pocos hablantes de las lenguas yumanas de la región. Así, se determinó que el kumiai cuenta con 40 hablantes, el paipai, con 60, el cucapá, 10 y del 
kiliwa, 5. Sin embargo, algunos estudios recientes no publicados indican que el número de hablantes es menor en el presente 2020.

Entretanto, Mena, Tinajero y Canett (2016), concluyeron que la ausencia de un sistema uniforme en la enseñanza del mixteco en una escuela indígena de Ensenada, ha dado como resultado un deficiente aprendizaje del idioma. Al respecto, Venegas y Julián (2015), encontraron formas heterogéneas en la enseñanza de la escritura del mixteco hacia los niños de esta comunidad indígena, que lejos de beneficiarlos los perjudica ampliamente, debido a que no hay una estandarización en la forma de enseñar la escritura de la lengua.

Finalmente, Espino (2015), señaló que existen familias zapotecas residentes en Ensenada con una dinámica de convivencia única y tradicionalista. La autora destacó tres puntos medulares del grupo en su identidad: la pertenencia a la comunidad de origen, la pertenencia paisanal y pertinencia-sociedad de destino, elementos importantes de la contextualización para el fortalecimiento de la identidad zapoteca.

Por lo anterior, este trabajo viene a cubrir un vacío de estudios exiguos sobre actitudes hacia las lenguas indígenas de migrantes en el ámbito bajacaliforniano, considerando que la dinámica de migración de los grupos indígenas es un fenómeno amplio, reciente y complejo. Por ello, el presente estudio tiene como objetivo identificar actitudes lingüísticas hacia el zapoteco y la construcción de su identidad por sus propios hablantes en la ciudad de Ensenada. En consonancia con el objetivo, se formularon las siguientes preguntas de investigación: ¿Cuáles son las actitudes hacia el zapoteco por hablantes de la lengua en la ciudad de Ensenada? ¿Cómo se representa la identidad de ser zapoteco en el proceso migratorio hacia Ensenada?

De este modo, el trabajo se articula con las siguientes secciones que seguirán a continuación: la descripción del espacio a donde arribaron los migrantes mixtecos; el marco teórico que sustenta la investigación; la definición de la forma en la que se estableció contacto con los informantes; el análisis de los datos recolectados; la discusión de los resultados; y las conclusiones.

\section{El nuevo escenario de llegada: Ensenada}


El Instituto Nacional de Estadística y Geografía, (Inegi, 2016) aseguró que los indígenas de Baja California representan alrededor de $1.45 \%$ de la población total del estado, el cual se divide en cinco municipios. En Ensenada los indígenas constituyen el $5.09 \%$; en Mexicali, el $0.63 \%$; en Playas de Rosarito, el $1.63 \%$; en Tecate, el $1 \%$; y en Tijuana, el 0.89\%. Si bien no se abordó la situación de los hablantes indígenas a nivel estatal, estos datos permiten comprender el estado actual en el cual se hallan las lenguas indígenas en la región.

Además, el mismo Inegi (2016), afirmó que el Estado de Baja California cuenta con una población total de 3315766 individuos. Acerca del municipio de Ensenada, espacio de la presente investigación, tiene una población de 486639 habitantes (Inegi, 2016). Es la tercera ciudad de la entidad con mayor población, después de Tijuana, que es la urbe con más habitantes y Mexicali, la capital estatal, pero es el municipio más grande del país por su extensión territorial. En este espacio, Ensenada, se encuentran diversas colonias de población indígena, particularmente el área de Maneadero, poblado que forma parte de la zona metropolitana ensenadense y la localidad de San Quintín, en el sur del municipio. En el aspecto económico, Ensenada se ha especializado en la producción agrícola, razón por la cual han arribado múltiples migrantes indígenas del centro y sur de México. En cuanto a la población indígena, se puede localizar en otras colonias de la ciudad y del municipio. Frente a su ubicación geográfica, es importante mencionar que el municipio de Ensenada no es totalmente fronterizo en términos territoriales, pues antes de él se encuentran los municipios de Playas de Rosarito y Tijuana, el cual se ubica en la zona de frontera. Sin embargo, la cercanía con los Estados Unidos ha generado un modo de vida de amplio contacto con el país vecino, por lo que se podría denominar a Ensenada como una población cuasi fronteriza. 


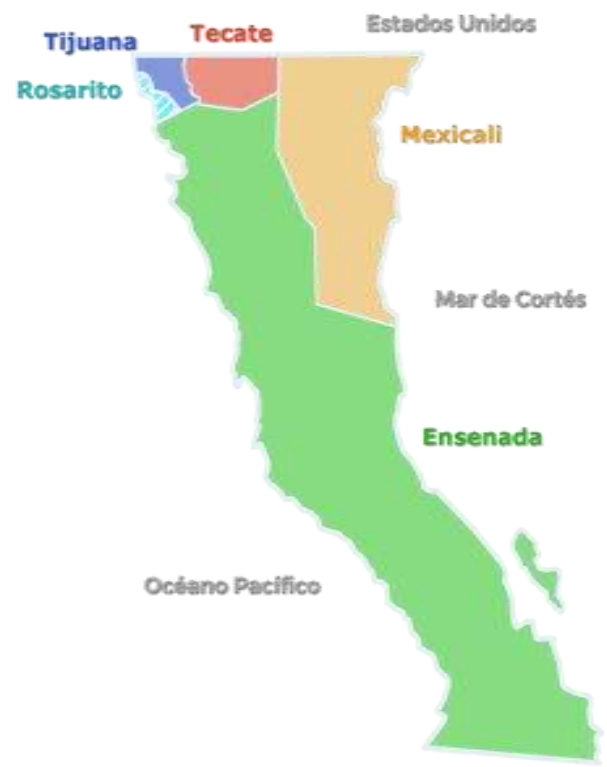

Figura 1. Mapa del estado de Baja California. Fuente: Baja California CENTER (s.f.).

Acerca del zapoteco, el grupo indígena que se analizó, se afirma que su lengua es la segunda más difundida en la región, con el 12.40 \% del total de hablantes indígenas de Baja California (Inegi, 2016a). Al igual que muchos grupos de población indígena, tales como mixtecos y triquis que predominan en la región, provienen del estado de Oaxaca, que actúan como población migrante tanto temporal o definitiva, es decir, algunos llegan y regresan a su lugar de origen o se trasladan a otros espacios, mientras que otros, toman la decisión de asentarse definitivamente en Baja California.

\section{La construcción de un marco teórico para el estudio de las actitudes linguiísticas y los aspectos migratorios}

Los estudios de actitudes lingüísticas corresponden a la sociolingüística, punto de partida para este trabajo (Fishman, 1979; Baker, 1992). Barriga y Parodi (1999) manifiestan que forma parte de la sociolingüística mexicana temas como el bilingüismo, la educación bilingüe-bicultural, el papel de la mujer y el multilingüismo, así como las actitudes de los hablantes indígenas hacia su lengua y el español. 
La forma de definir una actitud lingüística tiene relación con el escenario, el método y las técnicas; además, típicamente contienen tres componentes, los cuales también influyen en la forma de definir las actitudes lingüísticas. Cabe mencionar que de acuerdo a Blanco de Margo (1991), las actitudes tienen tres líneas de trabajo: 1. la variación lingüística, 2. los procesos psicológicos, sociales y culturales asociados al uso del idioma, 3. El comportamiento de hablantes frente a su lengua. Frente a esta directriz, este estudio se ubicaría en la posición 2, pues se abordó el proceso social de los hablantes frente al proceso migratorio y su identidad.

En este punto, se hará revisión de algunas definiciones de actitudes lingüísticas para orientar la construcción teórica del presente trabajo. Para Gardner (1985), las actitudes lingüísticas se entienden como una reacción evaluativa hacia un objeto o referencia que tiene su punto de partida en las creencias personales del hablante. De acuerdo con el autor, los sujetos son evaluadores de su entorno, pues tienen reacciones hacia otras personas, sus lenguas, su identidad y vestimenta, es decir, existe una interacción significativa entre el sujeto y su medio hacia el que se desencadenan reacciones, entre ellas las que se dirigen a las lenguas con las que convive. Por su parte, Moreno (1998) ofreció la siguiente definición:

Es una manifestación de la actitud social de los individuos distinguida por centrarse y referirse específicamente tanto a la lengua como al uso que de ella hacen en sociedad, y al hablar de «lengua», incluimos cualquier tipo de variedad lingüística: actitudes hacia estilos diferentes, dialectos diferentes o lenguas naturales diferentes (p. 179).

Esta perspectiva encaja con la investigación, ya que la actitud es consecuencia del ámbito social de los sujetos hacia una lengua, la cual tiene un uso en el medio, como se expresa en la definición, y por lo tanto, los hablantes tienen acciones hacia su idioma, lo cual se refleja en su mantenimiento, la transmisión hacia las nuevas generaciones o la asignación de funciones de la lengua en distintos ámbitos, pero también se encuentran los procesos adversos, como el desplazamiento y la extinción del idioma. Una tercera definición la provee Blanco de Margo (1991), quien describió a las actitudes lingüísticas como: 
[...] aquellos comportamientos subjetivos que el hablante tiene para con su lengua, que se manifiestan en general a través de creencias, prejuicios y valoraciones sobre su variedad lingüística, sobre otras lenguas o sobre determinados hechos del lenguaje. Tales juicios de valor se corresponden en algunos casos, con la realidad lingüística (como la defensa de un idioma que está siendo desplazado por otro en una situación de contacto) así como muchas veces están asociados a ideologías de algún signo (estéticas, religiosas, etc.) (p. 9).

Así, esta definición determina a las actitudes lingüísticas no solo como comportamientos o conductas asociadas a la subjetividad del hablante, sino que también profundiza en este último aspecto, como un conjunto de creencias, valoraciones y juicios orientados hacia la lengua. Tales aseveraciones pueden ser imaginarias, pero también hechos que se corresponden con la realidad lingüística para el hablante y la lengua. La definición, al ser una puerta que abre tanto los juicios como los valores de la lengua dentro de una realidad para el hablante, permite tratar el tema del presente trabajo, ya que es importante identificar lo que el hablante conoce, sabe y hace hacia su lengua en el contexto.

Por lo tanto, la definición de las actitudes lingüísticas, basada en la planteada por Blanco de Margo (1991), se ajustó a la propuesta por este estudio: las actitudes linguiísticas son consecuencia de la percepción del hablante frente a la realidad de la lengua, la cual genera saberes, afectos y conductas, a partir de diversos procesos sociales como el uso, el contacto con otras lenguas y la migración, la identidad, entre otros factores que afectan la situación de una lengua en un espacio y en un momento explícito, es decir, el lugar y el tiempo son determinantes para la modificación de las actitudes.

Las actitudes lingüísticas, como ya se dijo líneas arriba, tienen tres componentes: el cognitivo, el afectivo y el conductual. El primero se orienta hacia los saberes y conocimientos del idioma. El segundo trata de las emociones, afectos y evaluaciones hacia la lengua. El tercero consiste en las acciones y conductas hacia el idioma (Baker, 1992; Fishman, 1979). Cabe mencionar que los tres componentes pueden combinarse en las actitudes lingüísticas, como en el siguiente ejemplo de uno de los informantes del presente estudio: «Para vivir aquí es importante hablar español, es lo que dicen». Así, se demuestra que actúan juntos tanto el componente conductual, el cual se expresa a partir de la importancia de hablar español como un acto, como el componente cognitivo, que desarrolla la idea del saber de la gente en cuanto al uso del idioma en la ciudad. Acerca del método y las técnicas de las actitudes se tratarán con detalle en la siguiente sección. 
Referente a la identidad, se tomará la noción de Giménez (2009), quien explicó que se asocia a la cultura, como una organización social de significados, que actúan interiorizados en la persona a partir de esquemas y representaciones simbólicas dentro de un contexto histórico que se estructura socialmente. La cultura es una serie de significados y una distinción que actúa en el sujeto, el cual se localiza en un contexto y momento histórico. Por otro lado, la identidad no se puede definir como única, pues existe una individual y una colectiva, pero sin ser totalmente dicotómica. Para nuestro objetivo, conviene la colectiva, por el tipo de datos que se analizaron.

Las identidades colectivas carecen de una conciencia y una autorreflexión, a diferencia de las individuales (Giménez, 2009; Sciolla, 1983). Además, las identidades colectivas son capaces de diferenciarse de su entorno, definir sus límites, situarse en un campo y mantener en el tiempo el sentido de tal diferencia. Sin embargo, según Giménez (2009) la identidad no se define por los rasgos culturales de los sujetos. Lo que sucede, por ejemplo, es que un grupo étnico puede mantener sus fronteras, pero al mismo tiempo adoptar rasgos culturales de otro grupo; la lengua y la religión, y al mismo tiempo seguir distinguiéndose de los otros grupos.

En este sentido, la definición de Be (2011, p. 180) sobre identidad situada complementa la comprensión del problema. El autor explicó que se trata del «conjunto de representaciones y prácticas culturales dentro de contextos y situaciones concretas, en donde las personas tienen distintos recursos disponibles». Pero la identidad de las personas, de acuerdo a Brennies (1994) depende de las circunstancias de los sujetos, quienes finalmente eligen qué mostrar en el escenario.

Sobre la migración, se concibe como un proceso en el que se generan configuraciones sociales y demográficas de un espacio, a partir de los propios intereses de los sujetos para su desplazamiento desde su lugar de origen hacia un nuevo punto de llegada (Be, Ramírez, Salinas, Solís \& Corona, 2018). Además, los autores señalaron que junto con la movilidad de personas también se crea un movimiento de bienes, capitales, materiales, información, entre otros elementos, en los que se ubica a la lengua como uno de ellos. Por lo tanto, la migración se convierte en una actividad que conlleva a nuevas dimensiones de una lengua, creando y propiciando que permanezcan las actitudes ya establecidas o se modifiquen en un 
nuevo estado del mundo, debido a las presiones que experimentan sus hablantes (Terborg y García, 2011).

Así, considerando las características de nuestros sujetos de estudio, se puede indicar que las actitudes lingüísticas, tal como se expresó en la definición propuesta, son parte de un escenario y momento determinado, los cuales se rigen por procesos sociales y culturales, como el de la migración que orilla a las lenguas y a sus hablantes a diversas prácticas, entre ellas la identitaria, y que son detonadoras de saberes, afectos y conductas, que constituyen los tres componentes de las actitudes lingüísticas.

\section{La metodología para la obtención de los datos: el contacto con los informantes}

Inicialmente, cabe señalar que existen dos posiciones acerca de las actitudes lingüísticas en cuanto a su concepción y que afectan al procedimiento para la obtención de los datos (Solís, 2002). La primera es la mentalista, la cual dice que las actitudes están guardadas en la mente de los hablantes, por lo que sólo métodos conocidos como los indirectos pueden acercarnos a las actitudes, en este caso destacan las técnicas del matched-guise y el diferencial semántico. La segunda posición, la conductista, considera que las actitudes son observadas en el medio directamente, por lo que los métodos que se usan son conocidos como directos. Las técnicas usadas son la entrevista, la observación participativa o indirecta, cuestionarios y los experimentos de interacción entre hablantes. En esta problemática, ninguna de las técnicas es mejor que otra, pues son las necesidades de la investigación las que determinan cuáles técnicas se utilizan.

Para el caso del presente estudio, se trabajaron las técnicas de la postura conductista, ya que se aplicó el cuestionario sociolingüístico del Instituto Nacional de Lenguas Indígenas, (INALI, 2008), adaptado a Baja California, así como el diario de campo. El primer instrumento, el cuestionario, se usó para saber cómo funciona en el escenario de migrantes indígenas de Baja California, por lo cual muchas de sus preguntas se orientaron a las actitudes lingüísticas e implícitamente a la identidad y la migración. Además, el cuestionario se acercó al formato de la entrevista y permitió obtener respuestas que, si bien no siempre son profundas, son precisas y básicas para los propósitos de la investigación. 
Por otro lado, para corroborar lo que los hablantes compartieron en el cuestionario, se agregó el diario de campo, en el que se hicieron anotaciones de las observaciones indirectas y directas sobre el uso de la lengua dado por los informantes.

Cabe mencionar el porqué del zapoteco como grupo de estudio. Durante el diseño del trabajo de campo, se consideró la idea de trabajar con diversos grupos étnicos asentados en Ensenada. Sin embargo, una vez recolectada la información en 45 cuestionarios, se descubrió que los datos fueron recolectados de una familia zapoteca de 10 miembros, quienes por el tipo de respuestas que se verán en el análisis constituían perfectamente un estudio rico y suficiente para el presente estudio, por lo que se decidió delimitar el trabajo únicamente a dicho grupo de población migrante en la ciudad de Ensenada.

En la Tabla 1 se presentan los datos personales de los informantes por diversas variables. La familia zapoteca (Morales) fue abordada en las inmediaciones del Fraccionamiento los Encinos, una colonia del norte de la ciudad de Ensenada. Se acudió a ellos a partir de la técnica de bola de nieve, la cual consiste en localizar sujetos con las características requeridas de forma no probabilística y cuya localización se torna a veces difícil, (Alloatti, 2014). En el caso de este estudio, se les preguntó a los vecinos de las inmediaciones si conocían a personas que hablaran una lengua indígena, e inequívocamente señalaron el hogar de la familia Morales.

Todos los miembros de la familia afirmaron hablar zapoteco como primera lengua, y relataron que la razón principal por la que vinieron a Ensenada fue por lograr una mayor estabilidad económica, pues en su lugar de origen (San Blas, Oaxaca) hay pocos ingresos, por lo que se vieron obligados a trabajar en los campos agrícolas y a administrar un negocio propio de venta de comida.

Además, los hijos habían estudiado la preparatoria y la universidad, por lo que cuentan con empleos en diversas empresas. Como hablantes del zapoteco suelen hablar la lengua entre ellos y con otros paisanos conocidos de la colonia 89, además de otras colonias de la ciudad. A continuación, se presentará una tabla con información relevante de los participantes del estudio, a fin de tener datos sociolingüísticos de los hablantes. 


\begin{tabular}{|c|c|c|c|c|c|c|c|c|}
\hline No. & Nombre & Género & Edad & $\begin{array}{l}\text { Clase } \\
\text { social }\end{array}$ & Estudios & Actividad & $\begin{array}{c}\text { Lugar } \\
\text { de } \\
\text { origen }\end{array}$ & $\begin{array}{c}\text { Tiempo de vivir } \\
\text { en Ensenada y } \\
\text { colonia en la que } \\
\text { viven }\end{array}$ \\
\hline 1 & Eleazar & Masculino & 27 & Media & Licenciatura & Negocio familiar & $\begin{array}{l}\text { San } \\
\text { Blas }\end{array}$ & $\begin{array}{l}15 \text { años } \\
\text { Los Encinos }\end{array}$ \\
\hline 2 & Fabián & Masculino & 31 & Media & Licenciatura & Jefe de área laboral & $\begin{array}{l}\text { San } \\
\text { Blas }\end{array}$ & $\begin{array}{l}15 \text { años } \\
\text { Los Encinos }\end{array}$ \\
\hline 3 & Patsia & Femenino & 33 & Media & Maestría & Universidad & $\begin{array}{l}\text { San } \\
\text { Blas }\end{array}$ & $\begin{array}{l}13 \text { años } \\
\text { Los Encinos }\end{array}$ \\
\hline 4 & Armando & Masculino & 30 & Media & Licenciatura & Empresa familiar & $\begin{array}{l}\text { San } \\
\text { Blas }\end{array}$ & $\begin{array}{l}4 \text { años } \\
\text { Los Encinos }\end{array}$ \\
\hline 5 & Rosalba & Femenino & 50 & Media & Nada & Dueña de Negocio & $\begin{array}{l}\text { San } \\
\text { Blas }\end{array}$ & $\begin{array}{l}15 \text { años } \\
\text { Los Encinos }\end{array}$ \\
\hline 6 & Eleazar & Masculino & 57 & Media & Preparatoria & En su propio negocio & $\begin{array}{l}\text { San } \\
\text { Blas }\end{array}$ & $\begin{array}{l}15 \text { años } \\
\text { Los Encinos }\end{array}$ \\
\hline 7 & Elba & Femenino & 29 & Media & Preparatoria & En su propio negocio & $\begin{array}{l}\text { San } \\
\text { Blas }\end{array}$ & $\begin{array}{l}15 \text { años } \\
\text { Los Encinos }\end{array}$ \\
\hline 8 & $\begin{array}{l}\text { Juan } \\
\text { Carlos } \\
\end{array}$ & Masculino & 37 & Media & Licenciatura & Jefe de operaciones & $\begin{array}{l}\text { San } \\
\text { Blas }\end{array}$ & $\begin{array}{l}4 \text { años } \\
\text { Los Encinos }\end{array}$ \\
\hline 9 & Alberto & Masculino & 22 & Media & Licenciatura & $\begin{array}{l}\text { Nini (así se describió el } \\
\text { informante). Quiere } \\
\text { decir: una persona que } \\
\text { no se dedica a ninguna } \\
\text { actividad. }\end{array}$ & $\begin{array}{l}\text { San } \\
\text { Blas }\end{array}$ & $\begin{array}{l}4 \text { años } \\
\text { Los Encinos }\end{array}$ \\
\hline 10 & Sagrario & Femenino & 20 & Media & Preparatoria & Estudiante licenciatura & $\begin{array}{l}\text { San } \\
\text { Blas }\end{array}$ & $\begin{array}{l}2 \text { años } \\
\text { Los Encinos }\end{array}$ \\
\hline
\end{tabular}

Tabla 1. Información de referencia sobre los participantes del estudio

El cuestionario sociolingüístico está dividido en 29 preguntas, tanto de opción múltiple como de preguntas abiertas. Los temas que trata tienen relación con el aprendizaje de una primera y segunda lengua, la función del idioma en la familia y la comunidad, los usos de la lengua en la escuela, las actitudes hacia el idioma, los vínculos con los medios de comunicación, el nivel de vitalidad de la lengua en niños y jóvenes, la escritura de la lengua y su enseñanza.

Para cubrir el espacio asignado en el presente estudio, la indagación se centró en las preguntas tendientes a: 1) la importancia de hablar zapoteco en la familia, 2) la importancia de hablar el idioma en Ensenada, 3) la frecuencia de viajes fuera de Ensenada y el uso de la lengua, 4) la identidad que los hablantes afirman tener. Las preguntas completas las veremos en el análisis. Debido a que el cuestionario es extenso, no fue presentado en el anexo por falta de espacio, pero puede consultarse el original en la página del INALI (2008), ya que el instrumento se elaboró a partir de una adaptación al contexto bajacaliforniano, como mencionamos anteriormente. 
En cuanto al tratamiento de los datos, se establece que el análisis realizado fue de tipo cualitativo e inductivo (Hernández, Fernández \& Batpista, 2006), en el cual se consideraron las respuestas de los hablantes para formar categorías semánticas que, a partir de las coincidencias entre los testimonios, establecieran una categoría de análisis que recibe un nombre propuesto por los autores del presente trabajo y que se evidencia en cada subnumeración.

\section{Actitudes hacia la lengua en el hogar y en la ciudad de Ensenada}

Para dar razón de la primera pregunta de la investigación, planteada en la introducción, se tuvieron en cuenta las respuestas del cuestionario sociolingüístico dadas por los hablantes. Las siguientes subsecciones representan una categoría de análisis para cada indicador del cuestionario.

\subsection{Actitudes en el hogar}

La primera categoría resultante fue a partir de la pregunta: ¿Considera que para su familia es importante hablar la lengua zapoteca? Sí_No_iPor qué? Todos los participantes respondieron afirmativamente. Entre los testimonios proporcionados destacan los siguientes, que presentarán en la Tabla 2. Cada frase de los informantes fue enumerada con un corchete para sistematizar y retomar, según se requiera en el análisis. Asimismo, debajo de cada enunciado en paréntesis aparece la edad y género del informante:

\begin{tabular}{|l|l|}
\hline $\begin{array}{l}\text { Sí, porque así se transmite de generación en } \\
\text { generación, para que no se pierda la lengua } \\
\text { [1] (27, masculino). }\end{array}$ & $\begin{array}{l}\text { Sí, mantener viva la lengua zapoteca [2] } \\
\text { (31, femenino). }\end{array}$ \\
\hline $\begin{array}{l}\text { Sí, para que continúe hablándose por más } \\
\text { generaciones [3] (33, femenino). }\end{array}$ & $\begin{array}{l}\text { Sí, para que siga la tradición familiar [4] (50, } \\
\text { femenino). }\end{array}$ \\
\hline
\end{tabular}




\begin{tabular}{|l|l|}
\hline $\begin{array}{l}\text { Sí, para mantener la familia unida [5] } \\
\text { (57, masculino). }\end{array}$ & $\begin{array}{l}\text { Sí, porque se mantiene la lengua viva [6] } \\
(29, \text { femenino }) .\end{array}$ \\
\hline $\begin{array}{l}\text { Sí, porque es la lengua que les enseñaron a } \\
\text { mis abuelos [7] (37, masculino). }\end{array}$ & $\begin{array}{l}\text { Sí, porque es parte de su cultura [8] } \\
\text { (20, masculino). }\end{array}$ \\
\hline
\end{tabular}

Tabla 2. Respuestas de los informantes sobre la importancia del zapoteco

Todos los informantes coincidieron en que es relevante para la familia el uso de la lengua zapoteca. En ese sentido, el hogar se recrea como un espacio que salvaguarda el idioma, en el que se observan actitudes positivas hacia el idioma, predominando el componente conductual de las actitudes que explica las acciones hacia la lengua; así, los hablantes expresan su deseo para que la lengua se mantenga como lazo de cohesión de la identidad y la memoria familiar, eje que resalta constantemente en las respuestas de los Morales.

Así, los testimonios dejan ver un fuerte aprecio hacia el idioma, reiterando constantemente el origen familiar, pues como ellos dijeron, en la lengua zapoteca se hallan sus raíces y punto de origen: «es la lengua que les enseñaron a mis abuelos», según el testimonio 7. De este modo, en la lengua se observa, además, un tránsito del pasado hacia el futuro, pues en todas las respuestas se capta el deseo de seguir hablando zapoteco.

Entre los aspectos particulares observados en las respuestas de los participantes, se notaron las combinaciones entre los mismos componentes actitudinales. Así, en el primer testimonio: «Sí, porque así se transmite de generación en generación, para que no se pierda la lengua» [1]. La combinación se da entre el cognitivo y el conductual: la primera parte hace referencia a la transmisión de generación en generación, mientras que la segunda parte da cuenta de la consecuencia de esa transmisión, con lo cual se piensa que se evitará la perdida de la lengua. En ese sentido, es notoria la conciencia del hablante sobre la importancia de hablar el idioma en el hogar, pues eso permite conservarlo, ya que el hablante sabe que existe un riesgo para que lengua se pierda. Tal como lo manifestó 
Bermeo (2011): «El ámbito o dominio del hogar es el último en el que desaparece la lengua, por lo que resulta relevante conocer sobre la vitalidad en ese contexto» (p. 182).

En este estado del mundo, un escenario principal en donde ocurren acciones, presiones e intereses a favor y en contra del idioma (Terborg \& García, 2011) es el hogar. Los hablantes mismos consideraron que dicho espacio es el motor de la vitalidad del idioma, por lo que el componente afectivo actúa de forma implícita, pues aunque no se evalúe directamente con un calificador al idioma, se entiende que de forma subyacente el informante valora su lengua en el ámbito del desarrollo del hogar.

En la siguiente respuesta «para mantener viva la lengua zapoteca» [2], no solo el informante reconoció el deseo de mantener el idioma, sino que añadió una pasión por la viveza de la lengua zapoteca a la que llama «lengua», es decir, se reconoce en ella un idioma. Del mismo modo, las palabras del tercer informante «para que continúe hablándose por más generaciones» [3], se orientaron fuertemente hacia el componente conductual, al usar el verbo hablar, el cual se aplica para que las generaciones futuras transmitan la lengua.

En este punto, se puede plantear que una lengua no solo existe en el pasado como un saber, o en el presente como un hecho actual, pues el futuro del idioma es relevante y los informantes zapotecos que radican en Ensenada lo muestran en sus testimonios, ya que tienen interés real en que su idioma siga perviviendo en los siguientes grupos etarios de generaciones venideras.

En la misma continuidad temática, cuando el cuarto informante dijo «para que siga la tradición familiar» [4], se expresa una mayor informatividad, pues la lengua no solo es un vehículo de comunicación, sino también una tradición, y por lo tanto, el idioma se constituye en un saber. La actuación del componente cognitivo de las actitudes lingüísticas junto al componente conductual expresa el seguimiento, la continuidad, la vitalidad del idioma. El informante ve en la lengua una práctica histórica del grupo familiar. La familia del informante ha existido por la lengua y le ha proporcionado una identidad, lo que hace importante su continuidad no solo lingüística, sino también la parte tradicional.

Por otro lado, el zapoteco está fuertemente ligado a las tradiciones y prácticas del estado de Oaxaca, siendo la entidad un referente de la lengua junto con sus prácticas culturales, 
por lo que no es en balde que el hablante, al observar las relaciones entre el idioma y las costumbres haga esta vinculación, como lo aseguró Espino (2015), donde existe una fuerte pertenencia identitaria de los zapotecos que llegan a Ensenada con su lugar de origen.

Las respuestas de los dos siguientes informantes son similares a otras que se han analizado. En la contestación «Es la lengua que les enseñaron a mis abuelos» [7], es interesante notar que nuevamente el hablante reconoce al zapoteco como una lengua, y en este caso, su respuesta se vincula al componente cognitivo, el saber, es el idioma de sus familiares, siendo este el punto de partida y la justificación para argumentar la importancia del zapoteco en el núcleo familiar. Es necesario resaltar aquí que la tradición familiar se encuentra arraigada, ya que los propios hablantes son observadores de su medio familiar, en donde han recolectado la información del uso del idioma de sus ancestros, deseando que perviva en las siguientes generaciones. La última respuesta «es parte de su cultura» [8], se cree que va en consonancia con el vínculo entre las tradiciones y la lengua.

\subsection{Actitudes hacia la lengua en el nuevo espacio de llegada}

La siguiente serie de respuestas que proporcionaron los informantes fue su visión sobre la importancia de hablar el zapoteco en la ciudad de Ensenada. Los testimonios fueron obtenidos a partir de la pregunta del cuestionario sociolingüístico: ¿Para vivir aquí es importante hablar zapoteco? Sí_ No_ ¿Por qué?

En principio de cuentas, todos los informantes de la familia Morales lo negaron. Entre las causas principales se encuentra el uso del español. En la Tabla 3 puede notarse la concordancia de las respuestas dadas a la pregunta planteada:

\begin{tabular}{|l|l|}
\hline $\begin{array}{l}\text { Porque aquí muchas personas no lo } \\
\text { hablan, no entienden zapoteco [9] } \\
\text { (27, masculino). }\end{array}$ & $\begin{array}{l}\text { Porque se habla español más que } \\
\text { zapoteco [10] (31, masculino). }\end{array}$ \\
\hline $\begin{array}{l}\text { Porque se habla español [11] } \\
(33, \text { femenino). }\end{array}$ & $\begin{array}{l}\text { Porque la mayoría habla español [12] } \\
(50, \text { femenino }) .\end{array}$ \\
\hline
\end{tabular}




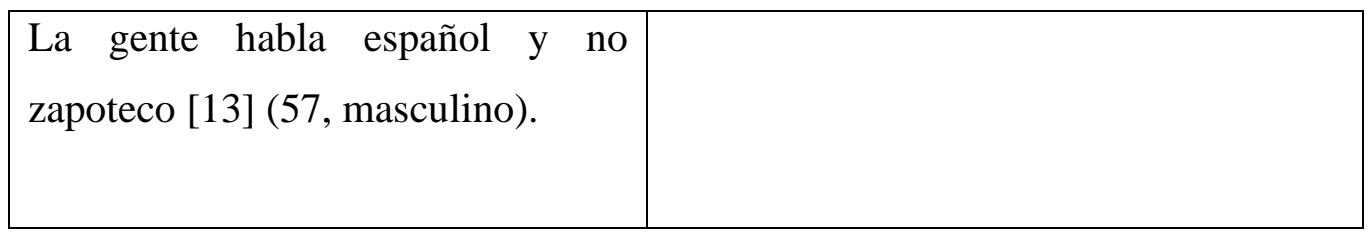

Tabla 3. Respuestas de los informantes sobre el uso del zapoteco

Los informantes del zapoteco reconocieron que en la ciudad de Ensenada el español tiene un uso predominante. En este aspecto, se nota un contraste entre su aprecio por el idioma étnico y su uso en los hogares frente al que se da fuera de los mismos. Los mismos son conscientes de la realidad lingüística y cómo opera el estado del mundo de la ciudad de Ensenada a favor del español. Desde los componentes de las actitudes lingüísticas, el elemento principal en las respuestas es el conductual por el uso del verbo hablar, pues con dicha acción se representa el uso del español.

Se hace evidente también la existencia de una dicotomía entre el «nosotros y los otros», pues los informantes hablan el idioma zapoteco en el hogar, son usuarios bilingües tanto del zapoteco como del español, según lo observado y registrado en el diario de campo. Sin embargo, solamente entre su núcleo familiar y cuando viajan a su lugar de origen los informantes dicen hablar la lengua. En tanto, en el mundo exterior a la familia, la ciudad de Ensenada, esta se decanta por el español, con algunas excepciones, pues los informantes comentaron que hablan zapoteco con otros que ellos llaman «paisanos», quienes viven en la colonia 89, cercana a Los Encinos, espacio donde ellos habitan. Cabe mencionar que también se registró en el diario de campo la mención acerca de no hablar zapoteco fuera del hogar en Ensenada, pero eso no los hace sentir mal, pues consideran normal para ellos el hecho de que en Ensenada se hable predominantemente el español.

\subsection{El espacio de origen y actitudes hacia la lengua}

Una siguiente pregunta del cuestionario sociolingüístico tiene relación con viajes externos. La cuestión que respondieron los participantes dice: ¿Qué tan seguido viaja fuera de la localidad? Seguido_ Poco_ Nada_a) Si viajas ¿a dónde viajas? b) ¿Utilizas el zapoteco ahí donde viajas? Sí_ No_ ¿Por qué? 
La frecuencia con la que respondieron los informantes fue la siguiente: ninguno respondió «Seguido»y «Nada», todos dijeron que lo hacen poco. Comentaron que viajan a Oaxaca y Oaxaca, San Blas, que es la ciudad de origen. Por otra parte, todos dijeron que sí usan el zapoteco en ese espacio de visita. Entre las razones que dieron destacan las que se muestran en la Tabla 4:

\begin{tabular}{|l|l|}
\hline $\begin{array}{l}\text { La mayoría de mi familia la habla } \\
{[14] \text { (27 masculino). }}\end{array}$ & $\begin{array}{l}\text { Es la lengua que se usa más ahí } \\
{[15](33, \text { femenino). }}\end{array}$ \\
\hline $\begin{array}{l}\text { Porque la mayoría habla zapoteco } \\
\text { allá [16] (30, masculino). }\end{array}$ & $\begin{array}{l}\text { Porque mi familia habla zapoteco } \\
{[17] \text { (50, femenino). }}\end{array}$ \\
\hline $\begin{array}{l}\text { Es la lengua de mi familia [18] } \\
\text { (57, masculino). }\end{array}$ & $\begin{array}{l}\text { Mi familia habla zapoteco ahí [19] } \\
(29, \text { femenino). }\end{array}$ \\
\hline $\begin{array}{l}\text { Tengo familia hablante de } \\
\text { zapoteco ahí [20] (37, masculino). }\end{array}$ & $\begin{array}{l}\text { Porque toda mi familia es zapoteca } \\
{[21] \text { (20, femenino). }}\end{array}$ \\
\hline
\end{tabular}

Tabla 4. Respuestas de los informantes sobre la frecuencia de uso del zapoteco

Las respuestas han dejado ver no solo la predominancia nuevamente del componente conductual, pues los participantes expresaron que la razón principal de hablar zapoteco en San Blas es que en ese espacio se usa la lengua y la familia de ellos son hablantes del idioma. Además, esta expresión es interesante por la apropiación discursiva acerca de las relaciones familiares, el espacio de origen y el hecho de ser zapoteco. Así, junto con el componente conductual, aparece el componente cognitivo, pues se enuncia un saber: el hecho de que la familia y, en San Blas, se habla el idioma zapoteco. La apropiación de la lengua zapoteca por parte de los hablantes es notoria cuando uno de ellos enunció la expresión «es la lengua de mi familia», (testimonio [18]). En este punto, se deja ver nuevamente el valor y la estrecha relación entre la familia con el zapoteco, lo cual genera 
una actitud que conlleva a afirmar que en el ámbito familiar el idioma tiene vitalidad. Además, los Morales son un claro ejemplo de una familia de migrantes zapotecos en la ciudad de Ensenada, que mantienen el vínculo con su lengua étnica y con el lugar de origen, San Blas, a través del idioma zapoteco.

\subsection{La identidad del ser zapoteco}

Para responder a la segunda pregunta de investigación planteada en la introducción, puede resumirse la importancia en los testimonios de la anterior categoría con la expresión «ahí y allá», que los informantes enunciaron en referencia a San Blas, el lugar donde se habla el zapoteco para los migrantes que se entrevistaron, lo cual genera un contraste entre la pregunta con la importancia de hablar el idioma en Ensenada, donde todos afirmaron que no se habla su lengua étnica, pues el español es el preferido de la población mayoritaria. «Ahí» viene a representar la comunidad en donde existe la conciencia del uso del idioma por parte de la familia. «Ahí», entonces, es el símbolo para la existencia y el presente del idioma para los informantes zapotecos, no tanto Ensenada para ellos, espacio en donde el español es el protagonista lingüístico principal.

La expresión de la informante con el testimonio [28]: «toda mi familia es de origen zapoteca», el cual aparece líneas abajo, muestra que además del valor expresado hacia el idioma, también se refleja una apropiación sobre el ser zapoteco. La identidad fluye también hacia la etnicidad, lo cual se expresa en los hablantes, quienes están afirmando el ser parte del grupo zapoteco. Así, en un espacio, Ensenada, en donde la mayor parte de la población habla español, pero en donde muchos grupos indígenas del sur de México han arribado, la identidad se encuentra, tal como lo dice Be (2011), de forma situada y contextualizada, en donde la temporalidad y el espacio juegan a favor de la formación de diversas actitudes y el mantenimiento de una identidad indígena.

Para complementar esta última información sobre cuestiones identitarias, se presentarán las respuestas de la pregunta ¿Usted se considera? Zapoteco_Indígena_Otro_ ¿Por qué? A la cual todos los informantes afirmaron ser zapotecos, esgrimiendo las causas que aparecen en la Tabla 5 . 


\begin{tabular}{|l|l|}
\hline $\begin{array}{l}\text { Porque nací ahí, en San Blas, Oaxaca } \\
\text { [22] (27, masculino). }\end{array}$ & $\begin{array}{l}\text { Porque nací en San Blas Oaxaca [23] } \\
(31, \text { masculino). }\end{array}$ \\
\hline $\begin{array}{l}\text { Mi familia es zapoteca [24] (33, } \\
\text { femenino). }\end{array}$ & $\begin{array}{l}\text { Por ser originario de San Blas, } \\
\text { Oaxaca [25] (30, femenino). }\end{array}$ \\
\hline $\begin{array}{l}\text { Es mi cultura [26] (50, femenino). } \\
\text { zapoteco [28] (29, femenino). }\end{array}$ & $\begin{array}{l}\text { Es la cultura de mi familia [27] (57, } \\
\text { femenino). }\end{array}$ \\
\hline
\end{tabular}

Tabla 5. Respuestas de los informantes sobre sus raíces culturales

En estos testimonios se dibuja la representación de ser zapoteco, tomando como causas el origen y nacimiento en San Blas, la familia como zapoteca y la cultura como propia o de la misma familia. En este caso, se aprecia la unión de la familia con el término zapoteco en el espacio de origen (San Blas), siendo que el ser zapoteco es una marca de identidad cultural que se lleva en la historia familiar. Los zapotecos, participantes del presente estudio, consideraron a San Blas como punto de partida, en donde se reencuentran con su ser y con su cultura, con su lengua y sus tradiciones, en donde han recibido las prácticas lingüísticas, tradiciones y un apellido étnico, el ser zapotecos. Así, de acuerdo con Espino (2015), la pertenencia al lugar de origen de los zapotecos de Ensenada hacia San Blas, es una forma en la que se representa su identidad étnica. Como complemento a esto último, el diario de campo registró emociones entusiastas que se desprenden del discurso de los informantes al referirse a San Blas, es decir, los zapotecos expresan con ahínco emociones cargadas de añoranza y pasión por su punto de partida.

\section{Algunas acotaciones}


Retomando las definiciones de actitud lingüística, así como la de identidad y migración que se consignaron líneas arriba en el marco teórico; la generación de saberes, afectos y conductas se reflejaron totalmente en las respuestas de los participantes del estudio, según la noción de actitud que se propuso. Las construcciones sociales de los informantes dadas a partir del contacto que tienen con el español en Ensenada, como consecuencia del proceso de migración que tuvieron desde su lugar de origen, los hace crear y formar un discurso para cada uno de los componentes de las actitudes lingüísticas analizadas.

El nuevo escenario, la ciudad de Ensenada, no está generando una modificación de sus actitudes hacia la lengua que constituya una amenaza para la continuidad del idioma zapoteco en el ámbito familiar. Al contrario, la lengua se representa como una marca de identidad en la que los aspectos culturales permiten a los hablantes de zapoteco sentir un distintivo de ellos mismos al considerar que son portadores lingüísticos de la lengua y sus representaciones culturales. Si bien Giménez (2009) trazó la identidad colectiva como una frontera abierta, entre nuestros participantes se observó que el zapoteco está ubicado como parte del patrimonio únicamente familiar, siguiendo una línea continua con la comunidad de origen.

Así, San Blas, se convierte en el espacio en donde se aviva, significa y resignifica la lengua y la identidad de ser zapoteco, el cual es tratado como un elemento histórico del origen familiar y donde las relaciones se socializan en zapoteco de forma natural. La añoranza de dicho lugar es el reforzador que crea una identidad situada hacia la lengua y el ser zapoteco. Se recrea en los informantes una situación de temporalidad hacia el pasado, presente y futuro, pues ven en San Blas un lugar que les permitirá seguir hablando su idioma y ser parte de una comunidad que se identifica como zapoteca. Así lo expresa Be (2011), con los migrantes mayas yucatecos quienes construyeron una relación especial con el espacio de procedencia al que nombran como «terruño».

Por otra parte, la identidad de los zapotecos juega un papel limitado en sus nuevas fronteras como grupo colectivo en Ensenada (Giménez, 2009). La frontera de ser zapoteco se restringe al hogar y al terruño, San Blas, en donde es posible seguir siendo zapoteco. Pero en Ensenada los hablantes han incorporado para sus prácticas cotidianas el uso del 
español y aunque en este estudio, por falta de espacio no se presentaron más datos de las funciones del español, los hablantes comparten modos de vida culturales que requieren del uso del español para las actividades cotidianas, tales como el ir de compras a San Diego, comprar en las segundas - tiendas que ofrecen productos con algún desperfecto, pero son nuevos, provienen desde Estados Unidos—, adquirir la comida en los mercados — que no son más que las tiendas de autoservicios, ya que en Ensenada, son casi inexistentes los mercados tal como se conocen en el centro y sur de México-. El mercado es el Walmart, Aurrera, Soriana, Calimax, el Florido, entre otras tiendas que reproducen los modos de vida metropolitanos, según mencionaron como experiencia los informantes, lo cual fue registrado en el diario de campo.

Para finalizar esta sección, la migración genera en los zapotecos de San Blas hacia Ensenada una configuración en la que se nota el arraigo hacia el ser zapoteco, pero con la conciencia de que el nuevo espacio ofrece el español para la mayor parte de las funciones comunicativas. Como se mencionó en el marco teórico, a partir de Be, Ramírez, Salinas, Solís y Corona (2018), la movilidad trae bienes tangibles e intangibles, por lo que el idioma zapoteco está presente en la familia a la que se estudió.

\section{Conclusiones}

La presente investigación encuentra actitudes altamente positivas por parte de los diez informantes zapotecos que respondieron el cuestionario sociolingüístico del INALI, adaptado al contexto bajacaliforniano. Sin embargo, hace falta cubrir a un mayor número de participantes no solo del zapoteco, sino también de otras lenguas indígenas de migrantes, a fin de conocer cómo el proceso migratorio influye en la vitalidad lingüística, qué funciones y usos del idioma se realizan y la configuración de la identidad étnica de los migrantes en espacios cuasi fronterizos como Ensenada. Como apunte, es importante resaltar que diversos estudios, por mencionar algunos (Sordo, 2019; Terborg \& Velázquez, 2008; Terborg \& García, 2011) han expuesto que los procesos migratorios son indicadores del desplazamiento lingüístico, en tanto en el presente trabajo la migración no ha sido un 
factor determinante para las actitudes positivas hacia el idioma zapoteco y su uso en el hogar, pero será pertinente en otro estudio profundizar en el mantenimiento de la lengua.

Las actitudes muestran una predominancia del componente conductual, ya que a partir del mismo los hablantes narran sus experiencias y visiones del uso del zapoteco y del español tanto en el hogar como en la ciudad. Asimismo, el lugar de origen (San Blas, Oaxaca) es el reforzador que permite estrechar relaciones con lo zapoteco, pues la familia y dicho espacio persisten en la memoria de los migrantes como el lugar anhelado en donde es posible a veces, volver a ser hablantes cotidianos del idioma. En tanto, en el nuevo escenario, ser zapoteco se configura como parte de una identidad colectiva, abierta y situada, producto de un proceso migratorio. Como dice Espino (2015), los zapotecos también generan una pertenencia en la sociedad-destino, es decir, se han adaptado a su nuevo espacio de llegada, incorporando las prácticas lingüísticas y sociales que se ejerce en la ciudad, sumándose a la vida cotidiana de la metrópoli.

Por otro lado, la ciudad de Ensenada ofrece diversas perspectivas de trabajo hacia los grupos, debido a su alta vocación en la producción de alimentos y bienes agrícolas, por lo que se presenta la oportunidad de analizar las constantes migraciones de indígenas que arriban a esta urbe, desde una visión antropológica y las diversas áreas linguiísticas interdisciplinarias.

\section{Referencias bibliográficas}

1. Álvarez, E. (2008). Estudio de actitudes lingüísticas maya-español a través del análisis del discurso en Holcá, Yucatán. (Tesis de maestría). Ciudad de México, Universidad Nacional Autónoma de México.

2. Alloatti, M. (2014). Una discusión sobre la técnica de bola de nieve a partir de la experiencia de investigación en migraciones internacionales. IV Encuentro Latinoamericano de Metodología de las Ciencias Sociales, 27 al 29 de agosto de 2014, Heredia, Costa Rica. La investigación social ante desafíos transnacionales: procesos globales, problemáticas emergentes y perspectivas de integración regional. En Memoria 
Académica.

Disponible

en:

http://www.memoria.fahce.unlp.edu.ar/trab_eventos/ev.8286/ev.8286.pdf

3. Baja California Center (s.f.). Baja California:

https://www.bccenter.mx/index.php/es/baja-california

4. Barriga, R. \& Parodi, C. (1999). Alfabetización de indígenas y política lingüística entre discurso. En A. Herzfeld \& Y. Lastra (Eds.), Las Causas Sociales de la Desaparición y del Mantenimiento de Lenguas en las Naciones de América (pp. 21-36). Hermosillo: Universidad de Sonora.

5. Baker, C. (1992). Attitudes and Language. Clevedon, Philadelphia, Adelaide: Multilingual Matters.

6. Be, P. (2011). Dimensiones culturales e identidades situadas: la herencia maya en migrantes yucatecos a Estados Unidos. Estudios de Cultura Maya, XXXVIII (otoñoinvierno), 167-192.

7. Be, P., Ramírez, E; Salinas, C., Solís, E. \& Corona, S. (2018). El sentido de pertenencia a través del espacio virtual: el carnaval poblano de Santa María Nenetzintla. Riti Journal, 12(6), 181-188.

8. Bermeo, V. (2011). La vitalidad del otomí en Santiago Mexquititlán, Querétaro. En R. Terborg \& L. García (Coords.), Muerte y vitalidad de las lenguas indígenas y las presiones sobre sus hablantes (pp. 177- 195). Ciudad de México: Universidad Nacional Autónoma de México.

9. Blanco de Margo, M. (1991). Lenguaje e identidad: actitudes lingüísticas en la Argentina 1800-1960. Bahía, Blanca, Argentina: Universidad Nacional del Sur.

10. Brennies, D. (1994). Foreword. En J. M. Bachnik \& C. J. Quinn, Jr. (Eds.), Situated meaning: inside and outside in Japanese self, society, and language. Princeton: Princeton University Press, IX-XII.

11. Castillo, (2007). Mismo mexicano pero diferente idioma: identidades y actitudes lingüísticas en los maseualmej de Cuetzalan. México: Universidad Nacional Autónoma de México.

12. Crhová, J. (2004). Actitud hacia la lengua. Mexicali: UABC-CEC-Museo; PACMYC (ICBC). 
13. Durán, J. \& Sauma, J. (2003). Actitudes hacia la enseñanza de la lengua maya en el municipio de Mérida, Yucatán. Un estudio desde la perspectiva de la Sociología del lenguaje (Tesis de licenciatura). Mérida, Universidad Autónoma de Yucatán.

14. Espino, D. (2015). Del Istmo de Tehuantepec a Baja California: experiencia migratoria y la reconstrucción de pertenencia en familias zapotecas en Ensenada. (Tesis de maestría). Xalapa: Centro de Investigaciones y estudios superiores en Antropología Social (Ciesas).

15. Fishman, J. (1979). Sociología del lenguaje. Madrid: Ediciones Cátedra.

16. Gardner, R. (1985). Social psychology and second language learning: The role of attitudes and motivation. London: Edward Arnold.

17. Giménez, G. (2009). Cultura, identidad y memoria. Materiales para una sociología de los procesos culturales en las franjas fronterizas. Frontera Norte, 41(21), 7-32.

18. Gooskens, C., Hilton, N., \& Anja, S. (2016). Is Swedish more beautiful than Danish? A matched-guise investigation. Linguistics, 53(2), 375-403.

19. Hernández, R., Fernández, C., Baptista, P. (2006). Metodología de la investigación. Ciudad de México: McGrawHill.

20. Hotařová, L. (2011). Matched-guise: la técnica de pares ocultos. Romanica Olomucensia, 23(1), 9-14.

21. Instituto Nacional de Lenguas Indígenas (2008). Cuestionario sociolingüístico básico. México: INALI. ms.

22. Instituto Nacional de Estadística y Geografía, Inegi, (2016). Panorama sociodemográfico de Baja California 2015. Aguas Calientes: Inegi.

23. Instituto Nacional de Estadística y Geografía, Inegi (2016a). Anuario estadístico y geográfico de Baja California 2016. Aguas Calientes: Gobierno del Estado de Baja California, Inegi.

24. Lambert, W. (1972). Language, Psychology, and Culture. Stanford: Stanford University Press.

25. Leyva, A. (2016). La configuración sociolingüística de las lenguas yumanas de Baja California. En M. Márquez Palazuelos, D. Toledo \& L. Márquez (Coords.), Experiencias en lenguas e investigación del siglo XXI (pp. 402-413). Mexicali: Universidad Autónoma de Baja California. 
26. Mena, Y., Tinajero, G. \& Canett, Z. (2016). Ya nos tienen todos revueltos. Revista Interacción Journal, 14(2014-2015/2015-2016), 51-68.

27. Moreno, F. (1998). Principios de Sociolingüística y Sociología del lenguaje. Barcelona: Ariel.

28. Pfeiler, B. (1993). La lealtad lingüística del indígena maya yucateco. Validación de la prueba del matched-guise. Estudios de Lingüística Aplicada, 11(17), 49-54.

29. Sánchez, M. \& Rojas-Berscia, L. (2016). Vitalidad lingüística de la lengua paipai de Santa Catarina, Baja California. Liames, 1, 157-183.

30. Sciolla, L. (1983). Identitá. Turín: Rosemberg \& Sellier.

31. Solís, M. (2002). The matched guise technique: a critical approximation to a classic test for formal measurement of Language attitudes, Noves SL. Revista de Sociolingüística. Teoria i metodologia Estiu: 1-6.

32. Song, Y. \& Wang, J. (2017). Actitudes lingüísticas hacia las variedades del español. Estudio empírico a partir de estudiantes universitarios de ELE en Pekín. Círculo de lingüística aplicada a la comunicación, 72, 201-216.

33. Sordo, J. (2019). Actitudes lingüísticas e identidad étnica en los estudiantes indígenas migrados a la zona metropolitana de Monterrey. Lengua y Migración, 11(1), 7-30.

34. Terborg, R \& Velázquez, V. (2008). La muerte de lenguas y la desventaja de ser nativo hablante del otomí en México. Universos, 5, 129-143.

35. Terborg, R. \& García, L. (2011). Las presiones que causan el desplazamientomantenimiento de las lenguas indígenas. La presentación de un modelo y su aplicación. En R. Terborg \& L. García (Coords.), Muerte y vitalidad de las lenguas indígenas y las presiones sobre sus hablantes (pp. 29-61). Ciudad de México: Universidad Nacional Autónoma de México.

36. Vargas, E. (2018). Actitudes lingüísticas de padres y madres en familias colombianas y mexicanas en Houston: un estudio cualitativo. Forma y Función, 1(31), 155-181.

37. Venegas, E. \& Julian, O. (2015). Un estudio de adquisición de la escritura del mixteco bajo, y su tratamiento didáctico en el nivel primaria de la ciudad de Ensenada, Baja California (Tesis de licenciatura). Mexicali: Universidad Autónoma de Baja California. 